\title{
The effect of cue familiarity on categorizing by preschool children
}

\author{
CAROL PATRICK and STUART I. OFFENBACH \\ Purdue University, West Lafayette, Indiana
}

\begin{abstract}
Two- and three-year-old children were administered a free-sorting task with familiar or unfamiliar forms and colors. We expected that children would be more likely to classify objects together when the objects matched in some way that was familiar to them. The results confirmed that expectancy. The results also indicated that experience with cues may override other factors, such as preference, in determining how a set of objects is sorted. We conclude that stimulus familiarity may be an important factor to consider in studies of the origins of children's classification abilities.
\end{abstract}

Formation of categories or classifications of objects or events gives a certain order to the complexity of the world. Accordingly, researchers have been interested in determining how children develop classification systems, as well as in the age at which this capability first appears. Much of this research has focused on three issues: (1) the rules children formulate to classify objects (Handel \& Rhodes, 1980; Rosch, Mervis, Gray, Johnson, \& Boyes-Braem, 1976); (2) whether similarity or complementary criteria are the bases for considering two stimuli as members of the same class (Denney, 1972, 1975); and (3) whether children use perceptual or conceptual features to define categories (Mori, Miyazaki, \& Kaku, 1980; Sigel, 1953). The literature also includes studies of the effects of selected stimulus characteristics, such as color and form (Gaines, 1970; Smiley \& Weir, 1966) on the development of children's classification systems. One variable that has not received much consideration thus far, stimulus familiarity, was examined in this study by determining whether it was easier for young children to categorize stimuli with familiar characteristics than to categorize stimuli with unfamiliar characteristics.

According to developmental theorists (e.g., Inhelder \& Piaget, 1964; Vygotsky, 1962), young (2 to 3 years old) children classify sets of objects according to complementary criteria, whereas older children classify on the basis of stimulus similarity. Complementary classifications occur when stimuli are grouped such that they form a unified whole (e.g., the child makes an elaborate design or builds a house out of a set of stimuli) or when items that generally appear together are placed in the same category (such as a hammer and a nail). Similarity classifications are those in which stimuli that are perceptually alike in some way, such as having the same shape or color, are grouped together or are subsumed under the same verbal label (e.g., furniture or people).

The authors' mailing address is: Department of Psychological Sciences, Purdue University, West Lafayette, Indiana 47907.
Inhelder and Piaget (1964) and Vygotsky (1962) attributed this developmental change to internal, organismic changes in the child's cognitive structures. Denney (1972, 1974; Denney \& Acito, 1974) found evidence for such changes, but attributed them to external environmental factors. Thus, Denney and Acito (1974) found that with special training, 2-and 3-year-olds were able to classify objects on the basis of similarity (only a few of these children classified using similarity without the training.

Denney (1974) argued that complementary classifications may be a more natural way to categorize stimuli. She proposed that complementary classifications may be more ecologically valid than similarity classifications (the latter occur primarily in educational or occupational settings). For example, in everyday settings, a hammer and a nail are seen together more often than a nail and a pin. In support of this hypothesis, both preschool children and older adults used complementary classifications in a free-sorting task (Denney, 1974). Since neither of these age groups was under educational or occupational pressures to focus on similarity, they may have been less familiar with the common components in the objects. Therefore, complementary objects were grouped together. These results suggest that differences in experience or familiarity with stimuli may be an important variable in determining whether classifications are made on the basis of complementary criteria or similarity criteria.

Another factor that complicates the study of classification is the possibility that children of different ages prefer different stimulus attributes. For example, there may be a shift in preference (or dominance or attention) during the preschool years from color to form (e.g., Brian \& Goodenough, 1929; Modreski \& Goss, 1969). Specific attention to one of these cues could interact with classification criteria to change or alter sorting behavior. Unfortunately, the existence of this developmental change is still unsubstantiated. The evidence 
for such a change has come from work by Brian and Goodenough (1929) and others (e.g., Corah, 1964; Suchman \& Trabasso, 1966); they reported that children younger than 3 years and older than 5 years preferrred form (the others preferred color). Modreski and Goss (1969) and Offenbach (1983; Offenbach, Baecher, \& White, 1973) failed to find the predicted developmental changes among 4- and 5-year-old children.

These shifts, when they are observed, also may reflect differences in experience or familiarity with the cues and dimensions. This possibility is consistent with findings reported by Gaines (1970) and by Tighe, Tighe, Waterhouse, and Vasta (1970) that preference for color or form could be altered by direct experience with the previously nonpreferred stimuli. In both studies, the experimental conditions could be viewed as increasing the children's familiarity with the preferred characteristic.

In view of the results of sorting and preference studies, we propose that individual differences in experience or in familiarity with stimulus characteristics may explain why some children classify stimuli on the basis of form and others use color. The purpose of the present study was to test that hypothesis with young preschool children.

\section{METHOD}

\section{Subjects}

The participants were 65 2-year-olds ( 35 boys and 30 girls; mean age $=2$ years 7 months) and 634 -year-olds ( 31 boys and 32 girls; mean age $=4$ years 6 months). The children were from preschools and daycare centers in two small Midwestern cities.

\section{Procedure}

Each child was assigned to one of four experimental groups before being administered a concept sorting task consisting of familiar (F) or unfamiliar (U) forms (f) or colors (c). The experimenter, who was seated across a low table from the child, administered the task individually to each child. Nine stimulus items, consisting of combinations of three colors and three forms, were arranged (in a predetermined order) in front of the child. The experimenter pointed to one (preselected) item, asked, "Can you find me more of these?", and gave the child an opportunity to pick up any stimuli thought to be the same as that exemplar. The familiar forms (Ff) were a circle, a triangle, and a square. The three unfamiliar forms (Uf) consisted of a sixsided irregular polygon, a 20-deg curved piece, and a six-sided figure with one rounded side (these forms are outlined in Figure 1). The familiar colors $(\mathrm{Fc})$ were highly saturated green, blue, and yellow, and the unfamiliar colors (Uc) were muted shades of raw umber, Mars violet, and burnt sienna. The four task conditions represented the factorial combinations of the dimensions [color (c) and form (f)] and familiarity [familiar (F) and unfamiliar (U)].

After the child stopped selecting stimuli, the experimenter asked, "Is that all of them?" or "Are there any more?" When the child indicated that there were no more objects like the exemplar, the session was ended and the child returned to the classroom.

\section{RESULTS}

The children's selections were categorized as being based on form or color (sorts not conforming to one of
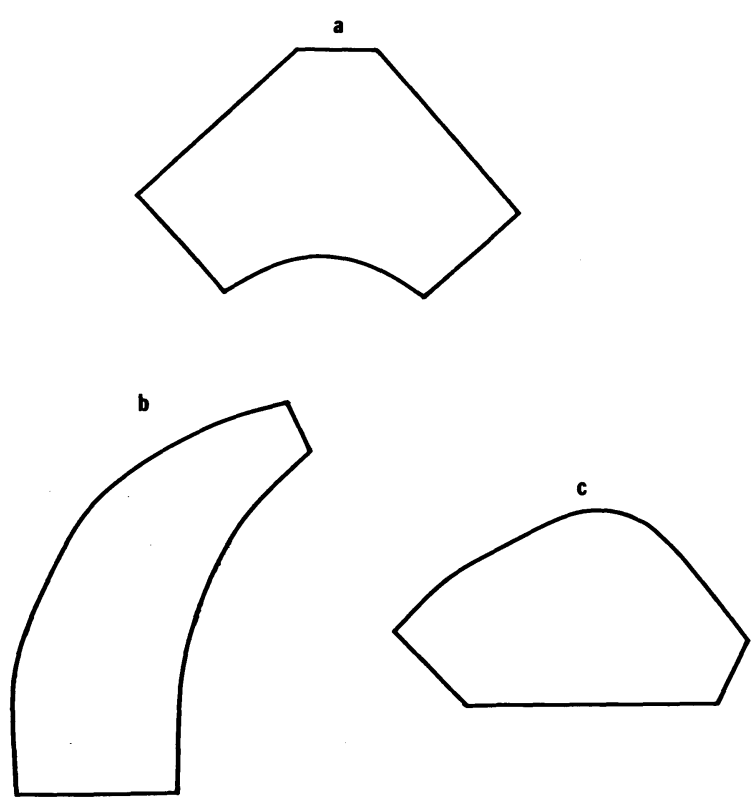

Figure 1. Three unfamiliar forms: (a) the six-sided irregular polygon with one concave curve; (b) the 20-deg curve; and (c) the six-sided polygon with a convex curve.

these rules were labeled unclassified). Most 4-year-olds responded by selecting only those items that matched the exemplar's form or color. Most 2-year-olds sorted by first choosing the stimuli that matched the exemplar's form or color, but then continuing to pick up items and adding them to the collection. These sorts were classified if the initially selected items conformed to the form or color rule (a few 2-year-olds' responses were classified when they picked up the items in a random order but placed those that matched the exemplar's form in one pile and the rest in another pile). The children whose sorts were unclassified had no apparent pattern to their selection of stimuli. Preliminary analyses indicated that there were no significant differences in sorting due to sex, and the data were pooled for the remaining analyses.

A chi-square analysis $(3 \times 4)$ was computed to compare the types of classifications made by the children at each grade level (Table 1). Overall, more matches were based on familiar cues than on unfamiliar cues $\left(\chi^{2}(6)=\right.$ 16.91 for age 2 years and $\chi^{2}(6)=14.76$ for age 4 years; $\mathrm{p}<.05$ in each case). Additional analyses indicated that both younger and older children selected items on the basis of form when the form was familiar (in Conditions FfFc and FfUc). In fact, no 2-year-old selected stimuli based on color when form was familiar. Both 2- and 4-year-old children selected stimuli based on familiar colors if the forms were unfamiliar (Condition FcUf).

Age differences in sorting also were observed when both forms and colors were unfamiliar (Condition UfUc). With these stimuli, as many 4-year-olds sorted using color as did using form. The 2-year-olds, how- 
Table 1

Number of Children Classifying Using Each Attribute

\begin{tabular}{lrrrr}
\hline Type of Sort & FfFc & FfUc & UfFc & UfUc \\
\hline & \multicolumn{2}{c}{2 Years Old } & & \\
Form & 9 & 7 & 3 & 1 \\
Color & 0 & 0 & 5 & 4 \\
Unclassified & 9 & 8 & 8 & 11 \\
& 4 Years Old & & \\
Form & 11 & 13 & 15 & 6 \\
Color & 3 & 3 & 10 & 7 \\
Unclassified & 1 & 0 & 0 & 2 \\
\hline
\end{tabular}

Note $-F=$ familiar $\boldsymbol{U}=$ unfamiliar $f=$ form $; c=$ color .

ever, either classified on the basis of color or did not classify the stimuli at all (the responses were unclassified). Another clear age difference was observed in the number of unclassifiable response protocols. Most ( 3 of 63) of the older children classified the items using one of the two attributes. The younger children, on the other hand, were far less efficient in classifying the stimuli; 36 of the 65 protocols were unclassifiable.

\section{DISCUSSION}

These results replicate earlier findings, but only when the form attribute was represented by familiar cues. When the form cues were unfamiliar and the colors were familiar, most children classified using the more familiar colors. Overall, both 4-year-olds and 2-year-olds classified stimulus objects on the basis of a familiar rather than an unfamiliar attribute, supporting our hypothesis that familiarity, not age, governs young preschool children's sorting behavior.

Denney (1972) theorized that experience with stimuli is a key factor in determining how children classify a group of stimuli. To the degree that familiarity and experience are equivalent constructs, our results support that conclusion. The present results also challenge the Piagetian developmental-shift hypothesis, according to which preschool children (especially younger ones) should choose all colors over all forms and that older children classify on the basis of form (Inhelder \& Piaget, 1964).

The present results also are consistent with the finding by Denney and Acito (1974) that although some 2-year-old children could classify similar stimuli together, most could not do so without special training. If these children are beginning to develop the ability to apply similarity classification rules, presenting them with cues that are not familiar might increase the problem difficulty to the point at which no consistent responses are possible. The finding that 11 of 15 younger children in the Condition UfUc failed to classify consistently supports this conclusion. The 4-year-old children continued to classify on the basis of similarity, regardless of familiarity; but the basis of their classifications shifted as familiarity shifted. Even at this age, however, there was some difficulty when both the cues of both attributes were unfamiliar. Classifications were not uniform, because both attributes were used.

One difficulty with the present study (and with others) is that we could not control the amount of experience each child actually had had with the attributes and cues used in the sort- ing task. If equally unfamiliar cues could have been found, controlled experience might have validated the effects observed in the present study. This problem does not invalidate the overall finding that children's early classification behavior is influenced by the ease and familiarity they have with the "tobe-classified" materials. Stimulus or attribute familiarity may be a vital link in our understanding of children's early classification behavior.

\section{REFERENCES}

Brian, C. R., \& Goodenough, F. L. The relative potency of color and form perception at various ages. Journal of Experimental Psychology, 1929, 12, 197-213.

Corah, N. L. Color and form in children's perceptual behavior. Perceptual and Motor Skills, 1964, 18, 313-316.

DenNey, N. W. Developmental study of free classification in children. Child Development, 1972, 32, 221-232.

DENNEY, N. W. Evidence for developmental changes in categorization criteria for children and adults. Human Development, 1974, 17, 41-53.

Denney, N. W., \& Acito, M. A. Classification training in twoand three-year-old children. Journal of Experimental Child Psychology, 1974, 17, 37-48.

GaInes, R. Children's selective attention to stimuli: Stage or set? Child Development, 1970, 41, 979-991.

HANDEL, S., \& Rhodes, J. W. Free classification: Element level and subgroup level similarity. Perception \& Psychophysics, 1980, 28, 249-259.

Inhelder, B., \& Piaget, J. [The early growth of logic in the child] (E. A. Lunzer \& D. Papert, Trans.). London: Routledge \& Kegan Paul, 1964.

Modreski, R. A., \& Goss, A. E. Young children's initial and changed names for form-color stimuli. Journal of Experimental Child Psychology, 1969, 8, 402-409.

Mori, T., MiYazaki, M., \& KaKU, N. Developmental changes in conceptual clustering in young children. Japanese Psychological Bulletin, 1980, 22, 1-6.

OfFenbach, S. I. The concept of dimension in research on children's learning. Monographs of the Society for Research in Child Development, 1983, 48(6, Serial No. 204).

Offenbach, S. I., BAecheR, R., \& White, M. Effect of method, instructions, and age on stimulus "preference" assessments. Journal of Genetic Psychology, 1973, 122, 253-261.

Rosch, E., Mervis, C. B., Gray, W. D., Johnson, D. M., \& Boyes-Braem, P. Basic objects in natural categories. Cognitive Psychology, 1976;8, 382-439.

Sigel, I. E. Developmental trends in the abstraction ability of children. Child Development, 1953, 24, 131-144.

SMILEY, S., \& WEIR, M. W. Role of dimensional dominance in reversal and nonreversal shift behavior. Journal of Experimental Child Psychology, 1966, 4, 296-307.

Suchman, R. G., \& Trabasso, T. Color and form preference in young children. Journal of Experimental Child Psychology, 1966, 3, 177-187.

Tighe, L. S., Tighe, T. J., Waterhouse, M. D., \& Vasta, R. Dimensional preference and discrimination shift learning in children. Child Development, 1970, 41, 737-746.

Vyootsky, L. S. [Thought and language] (E. Hanfmann \& G. Vakar, Eds. and Trans.). Cambridge, Mass: M.I.T. Press, 1962.

(Manuscript received July 11, 1983.) 